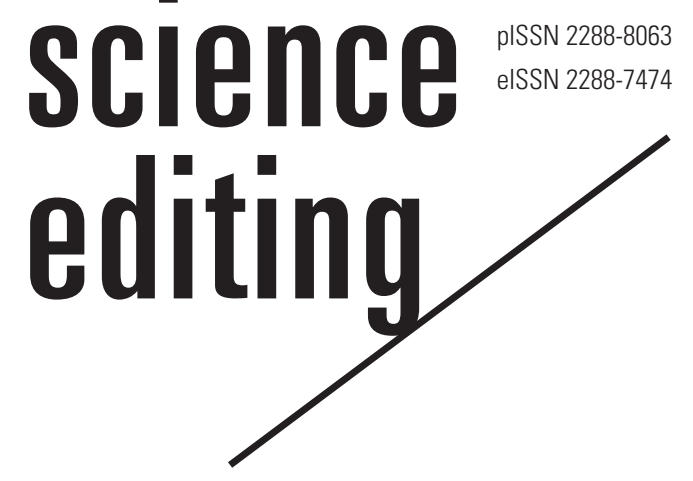

\title{
Preprint acceptance policies of Asian academic society journals in 2020
}

\author{
Ye Jin Choi $^{1 *}$, Hyung Wook Choi ${ }^{2 *}$, Soon Kim ${ }^{1}$ \\ ${ }^{1}$ Research Institute for Social Science, Ewha Womans University, Seoul, Korea; ${ }^{2}$ College of Computing and Informatics, Drexel
} University, Philadelphia, PA, USA

\section{Abstract}

Purpose: In the current era of the coronavirus disease 2019 pandemic, the trend of sharing new research results through preprint platforms is receiving more attention from researchers than ever before. Preprints have been recognized as a primary and essential method to disseminate new findings faster than traditional publications. Therefore, it has become necessary for journals and editors to acknowledge these changes, prepare preprint policies, and notify authors accordingly. This study aimed to review the status of preprint policies of international publishers and Asian academic society journals.

Methods: In total, 383 Asian academic society journals registered in Science Citation Index Expanded were selected as a dataset for analysis between December 11, 2020 and January 8, 2021. Three different parameters were investigated whether each journal had a preprint policy, whether journals allowed preprint manuscripts to be submitted, and whether preprint articles were allowed to be included in the references.

Results: Among the 383 Asian academic society journals from 22 countries, only 28 journals accepted preprint manuscripts, and eight allowed the use of preprint manuscripts as references. Japan had the most journals that both had preprint policies and accepted preprint manuscripts, with 13 journals, followed by Korea with 10 journals.

Conclusion: Despite the limitations of this study, the results show that editors and journal staff should understand the current preprint trend and try to prepare preprint policies that best meet

Keywords

Journal policy; Open science; Preprint; Preprint policy; Society journal

\section{Introduction}

Background/rationale: Most researchers generally do not share their studies with others until the studies are published. However, it can take at least a few months-and sometimes even years-before publication. To resolve this problem, in recent years, increasingly many re- 
searchers have used preprints, which allow them to upload studies online before peer review [1]. In particular, due to the coronavirus disease 2019 (COVID-19) pandemic, preprints have been recognized as a primary and essential research resource for the faster dissemination of results than is possible through traditional publications. This change has enhanced the position of preprints among researchers. On June 1, 2020, preprints accounted for one-quarter of all research output among COVID-19-related studies, including over 42,700 scholarly articles, 3,100 clinical trials, 420 dataset, 270 patents, 750 policy documents, and 150 grants [2].

Preprints are defined as drafts of research papers shared before peer review. Most preprints are given digital object identifiers (DOIs), which can be cited in other research papers. The advantages of preprints include enabling faster distribution, giving feedback opportunities, publishing research results that would be difficult to publish in traditional papers, strengthening research experience among young researchers, preventing the theft of research ideas, and expanding the accessibility of academic research [3].

Some aspects of why preprints are attracting attention warrant a closer examination. First, by publishing a draft paper containing research results as a preprint, ownership of the findings and credits can be secured. If there is a dispute over who first discovered a particular result, the work published as a preprint can be a permanent part of the academic record that can furnish solid proof of the dispute. For this reason, the National Institutes of Health and Wellcome Trust allow researchers to use preprint articles as references when applying for research funds [1].

Second, in the traditional system, a manuscript goes through the process of receiving feedback from two to three peer reviewers after submission, but posting the manuscript as a preprint allows readers to point out major flaws or errors. Furthermore, new research or data strengthening the author's argument may be proposed, and preprint publications can even lead to collaborations that can be published in more prestigious journals [1].

Third, a manuscript published as a preprint is not the final version of the research paper, but can be linked to the final published article through Crossref, thereby increasing the visibility and citation of the final published paper [1].

As such, researchers are becoming increasingly interested in preprints as a distribution channel of new research. In particular, more and more researchers are asking whether they can post their manuscripts on a preprint server before the official paper is submitted to an academic journal. Since authors usually refer to the authors' guidelines on a journal's homepage before submitting a paper, a detailed preprint policy should be clearly described on the journal homepage, specify- ing whether the journal considers manuscripts that have already been published as preprints, to reduce authors' confusion.

Related studies: Klebel et al. [4] investigated the preprint policy status of 171 major academic journals, including commercial ones. Of the 171 journals, 120 (70.2\%) allowed preprints. including 39.2\% that allowed preprints before peer review, while $22.8 \%$ did not have a preprint policy. In general, natural science journals tended to allow preprints only for the first submission before peer review.

Massey et al. [5] assessed the preprint policy of the 100 clinical journals with the highest impact factors. Most journals (86\%) allowed authors to submit articles previously posted as preprints, and 13 journals made this decision on a case-bycase basis.

Vuong [6] suggested that by allowing authors to share working papers, preprints, postprints, or published articles economically, preprints can be a valuable contribution as an open platform to share raw data with a wider audience.

Kirkham et al. [7] reviewed all biomedical preprint platforms to understand key characteristics and policies. Their results implied that authors now have the option to make their research publicly available at little or no cost and to take early ownership of their findings with the growing number of preprint platforms available in biomedical and medical research. Furthermore, on many preprint platforms, there are few limitations regarding authors' future ability to publish the research in the peer-reviewed journal of their choice.

Large commercial publishers with sufficient personnel and budget have been able to adapt to the preprint trend well; however, small academic society journals have found it difficult to apply preprint policies quickly. According to the COPE (Committee on Publication Ethics) discussion document released in 2018, a preprint was defined as a "scholarly manuscript posted by the author(s) in an openly accessible platform, usually before or in parallel with the peer review process" [8].

A manuscript posted on a preprint server can potentially share false results because it does not go through peer review, which is why some researchers are reluctant to publish a manuscript in preprint out of fear that journal editors may not accept the submission of the draft. However, the traditional academic publishing process is perceived by researchers as very time-consuming, and the advantages of preprint have recently been highlighted. Most of researchers' fears about preprints have been resolved because there are ethical and professional ways to deal with mistakes and errors at the academic level, and prominent journals such as Science and $\mathrm{Na}$ ture, as well as many open access journals, allow manuscripts published as preprints [3]. A study by Serghiou and Ioannidis 
science editing /

[9] found that both Altmetrics scores and citations were higher for articles that were originally published as preprints than for articles with no preprints. It has also been confirmed that preprint studies attract more attention from social media as well as traditional citations, which contributes to research influence at the author and article level.

With the proliferation of open science, the trend of sharing research results through preprints is increasing both in the field of energy physics and in various other fields where preprint culture was not originally popular [10]. Preprints are rapidly expanding to all academic fields for the prompt dissemination of research results. There is also a new publishing model that encourages journals to use AI programs to find and recommend the submission of appropriate papers from preprint servers [11].

Due to the ability of preprints to facilitate the rapid sharing of research results and to augment the influence of research results, the interest of researchers and research funding agencies in preprints is increasing. Therefore, it is necessary for academic societies that publish journals to pay attention to preprints and to clearly inform researchers about related preprint policies on the journal homepage. As such, the preprint policies of large international publishers and renowned academic journals were examined, as well as how small academic publishers are responding to them.

Sherpa Romeo's publication policy statistics showed that 1,064 (42\%) of 2,562 publishers allowed preprints and postprints for sharing [12]. Looking at the information required when entering new publisher policies on the Sherpa Romeo site, detailed policies can be entered regarding the linkage of preprints with final published articles [13].

Objectives: In this study, the preprint policies of large international publishers were checked based on Cho's study [14]. Based on these results, we investigated how Asian academic society journals are responding to preprint policies to suggest an appropriate preprint policy for Asian academic society journals.

\section{Methods}

Ethics statement: This study did not involve human subjects, so neither approval by the institutional review board nor obtainment of informed consent was required.

Study design: This bibliometric study based on journals' policies investigated Asian academic society journals preprint acceptance policies in light of the preprint policies of international publishers.

Background data: First, the contents of preprint policies were divided into international commercial publishers, mega open access journals, and prestigious society journals. The present status of preprint policies from Asian academic society journals was then analyzed. Specifically, the following preprint policies were investigated: whether the journals 1) allowed the acceptance of preprint manuscripts, 2) linked preprints to the final published articles, 3) allowed the use of preprint manuscripts as references, 4) or had other preprint policies that did not fall into above categories [14]. The full results are presented as supplementary materials.

Data sources/measurement: To collect data, between December 11, 2020 and January 8, 2021, 551 journals published by academic societies from Asia that were listed in the 2019 Science Citation Index Expanded were directly accessed through journals' websites to investigate the following parameters: 1 ) whether the journal had a preprint policy, 2) whether preprint manuscripts could be submitted, and 3) whether preprint articles could be included as references. As a result, 119 of 551 journals that were linked to commercial publishers were excluded from the analysis. Journals that did not provide information in English were excluded, as were journals for which the journal's official site could not be accessed due to errors, leading to the inclusion of 383 journals in the final dataset for analysis (Dataset 1).

In order to increase the reliability of the study, three researchers conducted a preliminary survey of 30 journals' websites to verify each other's findings and try to produce the same results. In addition, after completing the survey of 551 journals, three researchers examined each other's work for post-validation, and each person examined the contents of the journal again to verify that the survey was correct.

Bias: There was no bias in selecting the target journals. All eligible journals were selected.

Statistical methods: A descriptive analysis was done. There was no experimental analysis of data.

\section{Results}

Journal count per country and region

Table 1 presents a classification of the 383 journals according to the publishing countries and continents. The journals were published in 22 countries. The region of East Asia and Russia accounted for the most journals $(n=238)$, and Japan was the country that published the largest number of journals $(n=$ 102).

Presence of a preprint policy

Thirty of the 383 journals had preprint policies, whereas the other journals did not provide information on their websites. The academic journals with policies are listed in Table 2. In terms of the subject areas, the journals with preprint policies were mostly in the medical field, although journals on biolo- 
Table 1. Journal counts per country and region

\begin{tabular}{|c|c|c|}
\hline Region & Country & Count \\
\hline \multirow[t]{6}{*}{ East Asia and Russia } & Japan & 102 \\
\hline & Korea & 76 \\
\hline & China & 25 \\
\hline & Hong Kong & 14 \\
\hline & Taiwan & 11 \\
\hline & Russia & 10 \\
\hline \multirow[t]{4}{*}{ Southeast Asia } & Malaysia & 9 \\
\hline & Thailand & 6 \\
\hline & Philippines & 3 \\
\hline & Singapore & 3 \\
\hline \multirow[t]{5}{*}{ Southern Asia } & India & 33 \\
\hline & Pakistan & 12 \\
\hline & Bangladesh & 3 \\
\hline & Sri Lanka & 2 \\
\hline & Nepal & 1 \\
\hline \multirow[t]{7}{*}{ Western Asia } & Turkey & 44 \\
\hline & Iran & 20 \\
\hline & Israel & 3 \\
\hline & Kuwait & 2 \\
\hline & Saudi Arabia & 2 \\
\hline & Jordan & 1 \\
\hline & United Arab Emirates & 1 \\
\hline Total & 22 & 383 \\
\hline
\end{tabular}

gy, mathematics, space science, and computer science were also represented. The content of the policies could largely be divided into policies encouraging authors to post their manuscripts on preprint servers for the rapid dissemination of research results and policies banning authors from posting their manuscripts as preprints, which they considered to be duplicate submissions.

Some of these journals stated that they permitted the submission of manuscripts that are already posted on preprint servers; some others stated that they allowed authors to upload manuscripts that were submitted to the journals on preprint servers; and still others clarified that they would not allow the submission of duplicate studies to their journals. However, few of them provided detailed guidelines that authors could follow during the peer review process and after publication. A few journals described how they would handle preprint manuscripts leading to the publication of the final versions. For instance, Investigative and Clinical Urology recommended authors "to disclose it with DOI in the letter to the editor during the submission process." Plant Biotechnology clarified that "At the time of submission to this journal, authors must inform the journal via a cover letter that the manuscript has been posted to a preprint server."

Regarding the linkage between the final published article and the preprint, Plant Biotechnology journal stated that "Once the article has been published in its final form on the journal website, the preprint server must link to the article on the journal's website." The Journal of Poultry Science said, "Any version of a manuscript that has been revised in response to reviewers' comments, accepted for publication or published in the journal should not be posted on a preprint server. Instead, forward links to the published manuscript may be posted on the preprint server." The World Journal of Men's Health (WJMH) stated, "If the preprint is accepted for publication, authors are recommended to update the info at the preprint with a link to the published article in WJMH, including DOI at WJMH. It is strongly recommended that authors cite the article in WJMH instead of the preprint at their next submission to journals." These observations show that several journals try to systematically follow and manage the process of preprints and formal publishing.

\section{Preprint manuscript acceptance}

Of the total of 383 journals, 30 had policies about the submission of preprint manuscripts. Specifically, 28 journals stated that they considered preprint manuscripts, while two indicated that they did not. The journals that accepted preprint manuscripts are listed in Table 2.

Among 28 journals, 14 were related to medicine. Other subject areas included space science, biology, and agriculture. Japan was the country with the most journals that had preprint policies, with 13 journals, all of which accepted preprint articles, followed by Korea with 10 journals that both had preprint policies and accepted preprint manuscripts. Both India and Iran had two journals with preprint policies, but one of the journals from Iran banned the submission of preprint studies. Lastly, Pakistan, Russia, and Turkey had one journal with a preprint policy each; however, the journal from Pakistan did not allow preprint articles.

\section{Allowance of preprint manuscripts as references}

Among 383 journals, only 10 provided information on whether preprint manuscripts could be included in the references, even including some journals that did not provide any information about whether they allowed preprint submissions. Eight academic journals stated that it was acceptable to include preprints. The corresponding journals are listed in Table 3.

All eight journals that allowed the use of preprint manu- 


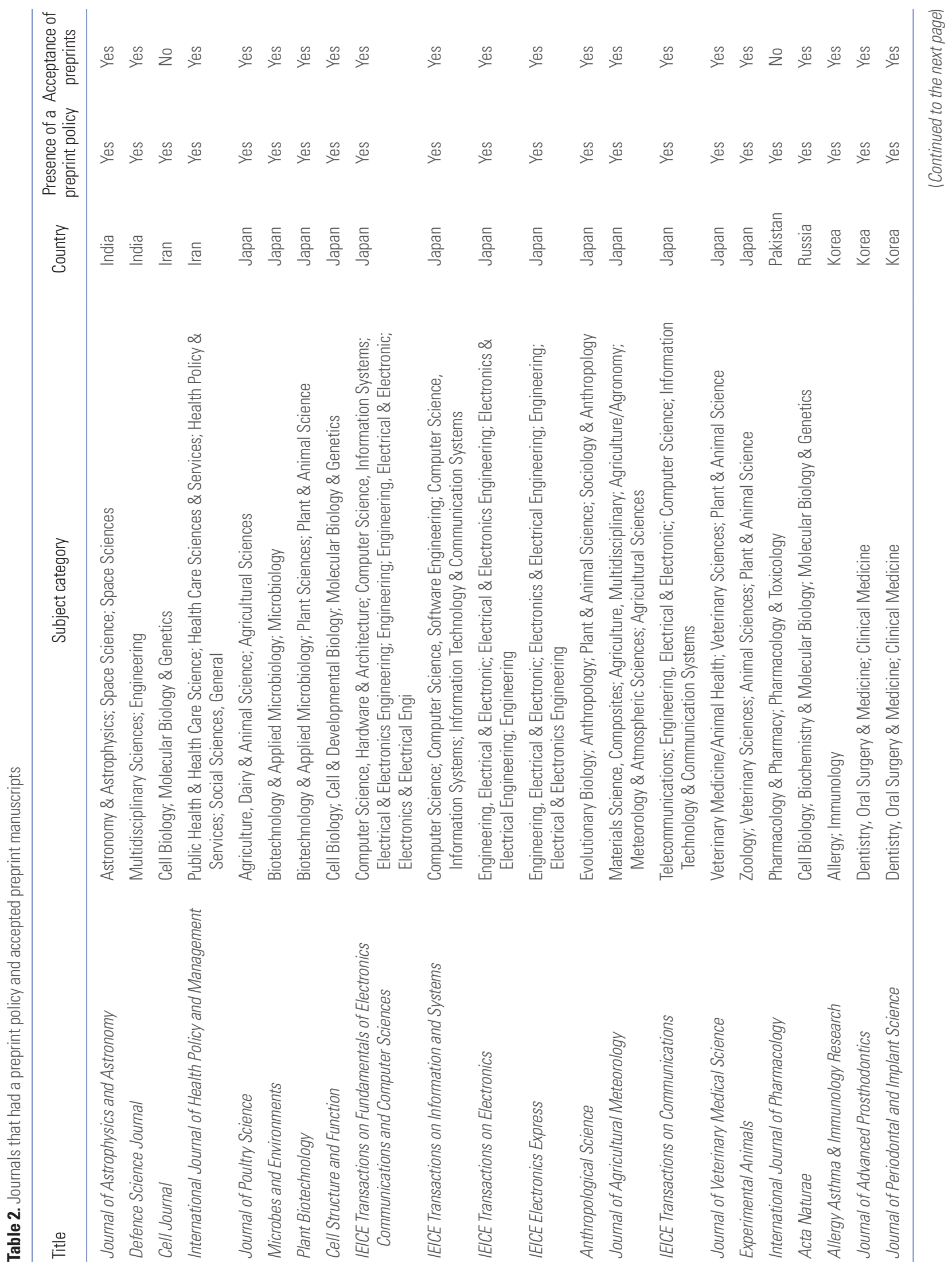


scripts in the references were science and engineering-related journals, distributed in various subject areas, including genetic engineering, public health, cell biology, space science, mathematics, and zoology. Japan had three journals, followed by Iran with two journals and China, Russia, and Taiwan with one each.

\section{Discussion}

Comparison with previous studies

Clear and easy-to-find preprint policies are important for authors to choose the right journal for publication. Whether a journal considers preprints as prior publications or not is an important factor for authors, many of whom would like to post preprints as a way to disseminate research rapidly.

Compared to the proportion of $7.3 \%$ of Asian academic society journals surveyed in this study, Klebel et al. found that the percentage of preprint acceptances among the top 171 journals was much higher, at $70.2 \%$. However, the majority of journals (57.3\%) did not clearly indicate whether preprints could be cited. In the journals that allowed preprint citations, $78 \%$ limited those citations to the references section, while $14 \%$ of journals restricted the citations of preprints to the text [4].

Preprint policies vary considerably from subject to subject. In the life sciences and earth science, $91 \%$ of all journals allowed pre-prints in any way, but this was only the case for $45 \%$ of journals in the humanities [4]. According to Massey et al. and Kirkham et al., the usage of preprints has increased [5,7]; however, the findings of this study are not comparable to those obtained for social science journals because the study only focused on SCIE journals. The result of this study based on Asian academic journals, showed that Korea and Japan had more journals that allowed preprints and more journals that specified preprint policies than other countries, but no significant differences in preprint policies were found across different subject areas.

\section{Suggestions for Asian society journal editors}

As academic journal editors, various details must be considered to determine the preprint policy. It is recommended that publication policies, including those related to preprints, should be clearly described and posted on the journal homepage, including whether a manuscript can be posted on a preprint server during peer review, whether to allow revisions of a preprint manuscript during the peer review process, and whether to refer to comments from preprint server during peer review. Moreover, if the journal uses a double-blind review process, preprint servers can cause difficulties because the author who posted the manuscript on the preprint server 


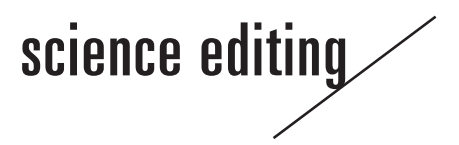

Table 3. Journals that allowed preprint articles in the references

\begin{tabular}{|c|c|c|}
\hline Title & Subject category & Country \\
\hline Bioimpacts & $\begin{array}{l}\text { Molecular Biology \& Genetics; Pharmacology/Toxicology; Pharmacology \& Pharmacy; } \\
\text { Medicine, Research \& Experimental; Pharmacology \& Toxicology }\end{array}$ & Iran \\
\hline International Journal of Health Policy and Management & $\begin{array}{l}\text { Public Health \& Health Care Science; Health Care Sciences \& Services; Health Policy \& } \\
\text { Services; Social Sciences, General }\end{array}$ & Iran \\
\hline Cell Structure and Function & Cell Biology; Cell \& Developmental Biology; Molecular Biology \& Genetics & Japan \\
\hline Journal of the Physical Society of Japan & Physics; Physics, Multidisciplinary & Japan \\
\hline Microbes and Environments & Biotechnology \& Applied Microbiology; Microbiology & Japan \\
\hline Research in Astronomy and Astrophysics & Astronomy \& Astrophysics; Space Science; Earth Sciences & China \\
\hline Markov Processes and Related Fields & Mathematics; Statistics \& Probability & Russia \\
\hline Zoological Studies & Zoology; Animal Sciences; Plant \& Animal Science & Taiwan \\
\hline
\end{tabular}

can be easily identified; this issue should also be carefully considered. Therefore, it is necessary for each author to clarify what was posted as a preprint in the cover letter and to provide a detailed description of the reference format of preprint manuscripts if the journal allows citations of preprint manuscripts [15].

It is also crucial to consider the issues of duplication of information and long-term digital preservation due to the increase in preprint usage in the long term. Discussions should also be held on how long or on what level versions of a manuscript should be preserved if an author posts a manuscript as a preprint version and in an institutional repository at the same time, as the information may overlap, potentially affecting management capabilities and technology infrastructure costs.

Unlike commercial publishers, academic journal editors do not have many ways to find out about the latest publishing trends, so it is important to attend journal editor meetings such as those held by the Korean Council of Science Editors to learn the latest information. Therefore, it is important for associations such as the Korean Council of Science Editors to provide information on the latest publishing trends on the association's website or through regular meetings to help editors acquire information on the latest publishing trends.

\section{Limitation}

Although the three authors double-checked each other's work, it is possible that the information on the websites may have changed or been updated since the research was conducted between December 11, 2020 and January 8, 2021. Therefore, more in-depth research is needed on the preparation of preprint policies, encompassing an analysis of the difficulties in developing a preprint policy through surveys or interviews with the editors of academic journals.

\section{Conclusion}

The study concluded that, out of a total of 383 society journals published in Asia listed in the 2019 Journal Citation Ranking, only $28(7.3 \%)$ indicated that they published preprint manuscripts. Only the author's guidelines and ethics policies were analyzed to determine preprint policies, so a limitation is posed by the possibility that society journals might have preprint policies that are not specified on their websites.

With the arguments over preprints in various fields of study, this paper can provide an opportunity for Asian journal editors to have more active discussions about how to follow global trends in preprint policies.

\section{Conflict of Interest}

No potential conflict of interest relevant to this article was reported.

\section{Funding}

The authors received no financial support for this article.

\section{Data Availability}

Dataset file is available from: the Harvard Dataverse at: https:// doi.org/10.7910/DVN/URPWLC

Dataset 1. Status of preprint acceptance of Asian academic society journals in 2020

\section{References}

1. Mudrak B. What are preprints, and how do they benefit authors? [Internet]. Durham, NC: American Journal Ex- 
perts; 2020 [cited 2020 Dec 13]. Available from: https:// www.aje.com/arc/benefits-of-preprints-for-researchers

2. Jackson A. How COVID-19 is changing research culture [Internet]. London: Digital Science; 2020 [cited 2020 Dec 14]. Available from: https://www.digital-science.com/blog/ news/new-digital-science-report-how-covid-19-is-changing-research-culture

3. Chiarelli A, Johnson R, Pinfield S, Richens E. Accelerating scholarly communication: the transformative role of preprints [Internet]. Geneva: Zenodo; 2019 [cited 2020 Dec 12]. http://doi.org/10.5281/zenodo.3357727

4. Klebel T, Reichmann S, Polka J, et al. Peer review and preprint policies are unclear at most major journals. PLoS One 2020;15:e0239518. https://doi.org/10.1371/journal. pone. 0239518

5. Massey DS, Opare MA, Wallach JD, Ross JS, Krumholz HM. Assessment of preprint policies of top-ranked clinical journals. JAMA Netw Open 2020;3:e2011127. https:// doi.org/10.1001/jamanetworkopen.2020.11127

6. Vuong QH. The rise of preprints and their value in social sciences and humanities. Sci Ed 2020;7:70-2. https://doi. org $/ 10.6087 / \mathrm{kcse} .193$

7. Kirkham JJ, Penfold NC, Murphy F, et al. Systematic examination of preprint platforms for use in the medical and biomedical sciences setting. BMJ Open 2020;10:e041849. https://doi.org/10.1136/bmjopen-2020-041849

8. Committee on Publication Ethics. Preprints [Internet].
London: Committee on Publication Ethics; 2018 [cited 2020 Dec 11]. Available from: https://publicationethics. org/node/38176

9. Serghiou S, Ioannidis JP. Altmetric scores, citations, and publication of studies posted as preprints. JAMA 2018;319:402-4. https://doi.org/10.1001/jama.2017.21168

10. Heuer RD, Holtkamp A, Mele S. Innovation in scholarly communication: vision and projects from high-energy physics. Inf Serv Use 2008;28:83-96. https://doi.org/10.3233/ISU2008-0570

11. Kim KH. Preprint status: focusing on science and engineering. Paper presented at: Korean Council of Science Editors 2020 Preconference workshop I; 2020 Jan 17; Seoul, Korea.

12. Kim JH. Preprint research trend. Paper presented at: Korean Council of Science Editors 2020 Preconference workshop I; 2020 Jan 17; Seoul, Korea.

13. Sherpa Romeo. Suggest a new publisher policy [Internet]. [place unknown]: Sherpa Romeo [cited 2020 Dec 10]. Availble from: https://forms.sherpa.ac.uk/new-publisher-policy. php?service-identifier=romeo 2

14. Cho HM. Preprint status: focusing on publisher and megajournal. Paper presented at: Korean Council of Science Editors 2020 Preconference workshop I; 2020 Jan 17; Seoul, Korea.

15. Yoon CH. Major preprint issues. Paper presented at: Korean Council of Science Editors 2020 Preconference workshop I; 2020 Jan 17; Seoul, Korea. 